\title{
A randomized, controlled, single-blinded, multicenter evaluation of the efficacy and safety of a once weekly two dose otic gel containing florfenicol, terbinafine and betamethasone administered for the treatment of canine otitis externa
}

S B King ${ }^{1}$, K P Doucette ${ }^{1}$, W Seewald ${ }^{2}$ and S L Forster ${ }^{3^{*}}$

\begin{abstract}
Background: Otitis externa is a common problem in small animal practice. Compliance with daily treatment is a major cause of treatment failure. The hypothesis tested is that a novel otic gel applied to the ear canal twice with a one-week interval is as efficacious as a daily otic suspension in the treatment of canine otitis externa. The study included 286 privately owned dogs with otitis externa.

In this single blinded randomized study, enrolled dogs received either an otic gel containing $1 \%$ florfenicol, $1 \%$ terbinafine and $0.1 \%$ betamethasone acetate twice with a one-week interval or a suspension containing hydrocortisone aceponate, miconazole and gentamicin daily for 5 days. Ears were cleaned with saline prior to administration of the first dose of medication. Dogs were evaluated at day (D) 0, 7, 28 and 56 with an otitis index score (OTIS-3), otic culture and cytology, pain and pruritus, and overall response to treatment (owner and investigator evaluation). Outcome measures were improvement of the OTIS-3 and number of dogs in clinical remission at each time point.
\end{abstract}

Results: OTIS-3 decreased significantly $(p<0.0001)$ by 63 and $64 \%$ for the otic gel and by 63 and $61 \%$ for the suspension on D28 and D56 respectively. There was no significant difference between groups at any time point with regard to clinical success, pain, pruritus, overall assessments or otic cytology and culture. The treatment response was considered excellent or good by approximately three quarters of both the clinicians and Owners. Otitis recurrence at D56 was seen in 11\% of both groups. Adverse events attributable to the ear medications were not noted.

Conclusions: Administering an otic gel twice at a one-week interval is an effective, safe and convenient way to treat canine otitis externa.

Keywords: Otitis externa, Otic gel, Clinical efficacy, Clinical safety, Florfenicol, Betamethasone, Terbinafine

\footnotetext{
* Correspondence: sophie.forster@elanco.com

${ }^{3}$ Elanco Animal Health Ltd., Lilly House, Priestley Road, Basingstoke,

Hampshire RG24 9NL, UK

Full list of author information is available at the end of the article
}

(c) The Author(s). 2018 Open Access This article is distributed under the terms of the Creative Commons Attribution 4.0 International License (http://creativecommons.org/licenses/by/4.0/), which permits unrestricted use, distribution, and reproduction in any medium, provided you give appropriate credit to the original author(s) and the source, provide a link to the Creative Commons license, and indicate if changes were made. The Creative Commons Public Domain Dedication waiver (http://creativecommons.org/publicdomain/zero/1.0/) applies to the data made available in this article, unless otherwise stated. 


\section{Background}

Otitis externa is one of the more common presenting complaints in small animal practice [1]. The inflammation of the external auditory canal may be due to a number of causes such as hypersensitivities, endocrinopathies, parasites and foreign bodies [2,3]. More than $50 \%$ of patients with atopic dermatitis exhibit otitis externa [4]. Predisposing factors include swimming, other causes of increased moisture in the ear canal or conformational factors such as pendulous pinnae $[1,3]$. The consequence of the resultant inflammation and associated decreased ear canal lumen is almost invariably an infection with bacteria and/or yeast organisms [3, 5]. Most topical ear medications on the market therefore contain a combination of antibiotic, antimycotic and anti-inflammatory agents, typically administered once daily [6]. Such products are typically packaged in multi-dose presentations, increasing the potential risk of cross-contamination between ears. In addition to the lack of diagnosis and treatment of the underlying disease, the other major problem treating dogs with otitis is owner and patient compliance [7, 8]. Administration of ear medications into a swollen and often painful auditory canal is a procedure frequently disliked equally by the owner administering the medication and the patient receiving it [8]. In a recent study, formulations requiring infrequent administration and ear cleaning improved the overall Quality of Life for pet owners and their dogs to a greater extent than those requiring daily administration [9].

Increasingly, multi-resistant bacteria make treatment of infections in veterinary medicine more difficult [10-13]. One of the factors recognized to increase the risk for development of such multi-resistant bacterial isolates is repeated exposure to low concentrations of antibiotics $[14,15]$, such as may occur with poor compliance with administration of a topical antibiotic. Ear medication that reliably maintains an above therapeutic concentration of active ingredients in the ear canal without relying on daily administration by the pet owner could thus be of great benefit to both dogs with otitis externa and their owners.

The study reported here compared the clinical efficacy and safety for otitis externa of an otic gel formulated in a single-use tube administered twice at a one-week interval with a more typical otic suspension administered daily as per the manufacturer's recommendation, with the objective of demonstrating that the otic gel was non-inferior to the suspension.

\section{Methods}

\section{Study design}

In this randomized, single-blinded, positive-controlled, multicenter field study, dogs received either a viscous otic gel (Osurnia ${ }^{\text {тм}}$, Elanco Animal Health, Greenfield, IN, USA) twice with a 1 week interval or an otic suspension (Easotic $^{\oplus}$, Virbac, Carros, France) daily for 5 days after an initial ear cleaning. Dogs were reevaluated at days 7, 28 and 56 after inclusion. The study was performed in accordance with the VICH GL9 (Good Clinical Practices). This manuscript was prepared after consultation of the checklist of the extension of the CONSORT statement for reporting of non-inferiority trials (www.consort-statement.org/extensions/overview/non-inferiority-and-equivalence-trials; page last accessed October 01, 2018).

\section{Study objects}

Dogs with clinical signs of otitis externa, an otitis index score (OTIS-3) of at least 5 and cytologic evidence of bacteria or yeast were included in the study.

\section{Inclusion, exclusion and withdrawal criteria}

To be eligible for inclusion, dogs were required to be a minimum of 8 weeks of age, of any breed, weight, sex or neuter status. They were excluded due to otic foreign bodies or parasites, if intended for breeding, or if they had been treated with either systemic or topical antimicrobial/ antifungals, ciclosporin or anti-histamines within the last 2 weeks, with ear cleaners or analgesic agents within the last week. Cases treated with systemic or topical antiinflammatories (i.e. corticosteroids or Non-Steroidal Antiinflammatory Drugs) within the last 28 days were not eligible for inclusion. Staff-owned or animals enrolled in other clinical studies within previous 3 months were not eligible for inclusion.

Similarly, dogs were not permitted into the study if they showed clinical signs of diseases which would interfere with the evaluation of the response. Lastly dogs in which the tympanum was ruptured or still not visible after an initial ear cleaning were also not included in the study. Withdrawal during the study occurred with adverse events that required intervention that could impact the study evaluation, lack of efficacy, administration of prohibited concomitant therapy, owner compliance or any other documented reason.

All study participants were required to sign an Owner Informed Consent prior to enrollment.

\section{Randomization and blinding}

Dogs were randomly allocated to the two treatment groups in a 1:1 ratio in blocks of four using the SAS/STAT ${ }^{\circ}$ procedure PLAN (SAS, Cary, NC, USA). A separate randomization list was prepared by the statistician for each participating center and provided to the dispensers in the form of sealed numbered envelopes, each containing the treatment allocation according to order of inclusion.

Due to the obvious difference in treatment protocols, Owner blinding was not possible. In each center, a designated person, the "dispenser", was responsible for the allocation of the dogs to the treatment groups and the administration of the treatments on D0 (and in the group 
treated twice with otic gel additionally on D7). Prior to each examination by the clinician, the dispenser recorded Owner answers regarding the clinical response, adverse effects, concurrent medications and other clinically relevant information, confirmed the Owner compliance, and prepared the Owner for the visit with the examining clinicians, to assure the continued blinding of the latter and gave relevant discharge instructions.

\section{Intervention}

Prior to inclusion, ear swabs were taken from each affected ear to collect material for culture and cytology. Subsequently, all dogs had their ears cleaned with physiologic saline to remove otic debris and permit visual evaluation of the tympana. Briefly this procedure consisted of filling the ear with warm saline solution, massaging the ear canal and wiping the entrance to the canal out with cotton wool. Dogs could be sedated for ear cleaning, if the ear was too painful to allow cleaning while conscious. Before discharge, each affected ear of dogs in the otic gel group received final formulation of $1 \mathrm{ml}$ of a viscous gel (Osurnia, Elanco Animal Health, Greenfield, IN, USA) containing $1 \%$ florfenicol, $1 \%$ terbinafine and $0.1 \%$ betamethasone acetate. Affected ears of dogs in the control group were treated with one pump (delivering $1 \mathrm{ml}$ ) of a suspension (Easotic, Virbac, Carros, France) containing $1.11 \mathrm{mg} / \mathrm{ml}$ hydrocortisone aceponate, $15.1 \mathrm{mg} / \mathrm{ml} \mathrm{micon-}$ azole and 1505 I.U./mL of gentamicin. Owners of dogs in the control group were sent home with instructions to administer the otic suspension once daily for four more days. Dogs were reevaluated after 7 days and dogs in the otic gel group were treated again. Ear cleaning was not repeated in either group at D7. These treatment protocols are in accordance with the package inserts for the two products.

\section{Clinical evaluation}

Prior to cleaning of the ears on D0, an otoscopic examination was performed and the OTIS-3 determined. The total score was the sum of the scores for erythema, edema/swelling, erosion/ulceration and exudate, each graded between 0 and 3 (total range 0 to 12) [16]. In bilateral cases, the ear with the higher score was selected to be evaluated throughout for all parameters. If both ears had the same score, the right ear was evaluated throughout. OTIS-3 was determined without further ear cleaning, on D7, D28 and D56.

Overall assessments of response to treatment were performed by the Owners and Investigators at D7, D28 and D56 (excellent, good, moderate or poor; see Table 1). Investigators evaluated pain at each visit using a Numerical Rating Scale ranged 0-3 (0, none; 1 , not painful on palpation but spontaneous head shaking; 2, not painful when pinna is raised but painful on palpation of base of ear; 3 ,
Table 1 Investigator and Owner Overall Assessment Scales

\begin{tabular}{|c|c|c|}
\hline & Investigator & Owner \\
\hline Excellent & $\begin{array}{l}\text { Clinical signs of the ear } \\
\text { evaluated during the first } \\
\text { examination have completely } \\
\text { disappeared }\end{array}$ & $\begin{array}{l}\text { My dog's ear condition has } \\
\text { completely recovered as } \\
\text { compared to before } \\
\text { treatment }\end{array}$ \\
\hline Good & $\begin{array}{l}\text { Clear amelioration of the } \\
\text { clinical signs of the ear } \\
\text { evaluated compared to initial } \\
\text { examination }\end{array}$ & $\begin{array}{l}\text { My dog's ear condition has } \\
\text { clearly improved compared } \\
\text { to before treatment }\end{array}$ \\
\hline Moderate & $\begin{array}{l}\text { Slight amelioration of the } \\
\text { clinical signs of the ear } \\
\text { evaluated compared to initial } \\
\text { examination }\end{array}$ & $\begin{array}{l}\text { My dog's ear condition has } \\
\text { responded only slightly to } \\
\text { treatment }\end{array}$ \\
\hline Poor & $\begin{array}{l}\text { Worsening or no change of } \\
\text { the clinical signs of the ear } \\
\text { evaluated compared to the } \\
\text { initial examination }\end{array}$ & $\begin{array}{l}\text { My dog's ear condition has } \\
\text { deteriorated or not changed } \\
\text { compared to before } \\
\text { treatment }\end{array}$ \\
\hline
\end{tabular}

painful when pinna is raised). Owners evaluated pain at each visit using a Visual Analog Scale (VAS) with the leftmost end marked 'The ear is not painful' and the rightmost end marked 'The ear is extremely painful'. Pruritus was assessed by Owners at each visit using a VAS with the leftmost corner underlined by the statement "The ear is not itchy" and the rightmost end with the statement "The ear is very itchy all the time'.

\section{Microbiological evaluation}

At D0, slides were prepared for in-house cytology using each clinic's standard staining technique. These were examined under low magnification to find an area of interest, with oil then applied and the area was evaluated for presence of microorganisms and inflammatory cells. If the cytology revealed bacteria or yeast, further samples for cytology and culture were sent to a central laboratory (Idexx Laboratories, Ludwigsburg, Germany). Presence of yeast, bacteria and neutrophils was evaluated semi-quantitatively. For neutrophils, a score of 1 was assigned where no neutrophils were seen, 2 for $1-10,3$ for $11-20$ and 4 for $>20$ within five microscopic fields under $\times 600$ magnification. For yeasts and bacteria, a score of 1 was given when no organisms were seen, few 2 (yeasts $<2$, bacteria $<5$ ), moderate 3 (yeasts 3-4, bacteria 5-25) and a high number of organisms recorded as 4 (yeasts $>5$, bacteria $>25$ ) based on five microscopic fields under $\times 600$ magnification.

On D28 and D56 additional specimens were obtained for cytology and culture and sent to the central laboratory.

\section{Minimum inhibitory concentrations}

All bacterial and fungal isolates from ear swabs taken both before and after treatment (D28 and D56) from cases treated with the otic gel were supplied to a microbiological testing laboratory (Don Whitley Scientific, Shipley, United Kingdom). The minimum inhibitory 
concentrations $\left(\mathrm{MIC}_{50 / 90}\right)$ against all bacterial and fungal isolates for florfenicol and terbinafine was determined using standardized broth microbiology as described by the Clinical and Laboratory Standards Institute (CLSI). Isolates of Staphylococcus pseudintermedius and Staphylococcus aureus were also screened for methicillin resistance.

\section{Outcome measures for efficacy}

The primary outcome measure was the percentage reduction in OTIS-3 at D28 compared to D0. Secondary outcome measures included the percentage of dogs with an OTIS- $3 \leq 3$ (considered a clinical success) at D28 and D56; the percentage reduction in OTIS-3 at D56 compared to baseline; the overall assessments by the Owners and Investigators at D28 and D56; the number of dogs with an OTIS- $3 \geq 5$ by D56 (considered relapses if the score at D28 was $\leq 3$ ); the decrease of bacterial or fungal counts on cytology at D28 and D56; the frequency (percentage) of dogs with either a bacteriological or fungal response at D28 or D56 (defined as absence of the microorganism isolated at D0); decrease in pain assessed by the Investigator at D28 and D56; decrease in pain and pruritus assessed by the Owner at D28 and D56; and the speed of response assessed by reduction in OTIS-3 at D7.

\section{Statistics}

An intention to treat (ITT) analysis with the last observation carried forward was performed. Patients completing the study without major protocol deviations were included in a secondary per protocol analysis (PP). Group means were compared between the two groups using an analysis of covariance (ANCOVA) model with the treatment group and baseline response as model effects. The influence of covariates was assessed by a second set of ANCOVA models with body weight, exudate type, chronicity, recurrence, duration, prior treatment, and type of infection as model effects. All calculations were performed using SAS Version 9.2.2, $\mathrm{SAS}^{\bullet}$ Prox Mixed and SAS Proc Glimmix were used for the ANCOVA and the GLM models (SAS, Cary, NC, USA).

The mean and standard deviation (SD) of the percentage improvement in a previous unpublished pilot study was 66 and 28 respectively. With a non-inferiority margin of $15 \%$ (which was considered clinically relevant) and a minimal power of $80 \%$, the appropriate number of study objects was calculated at 100 dogs per group.

\section{Safety evaluation}

Clinical safety of the product was evaluated through the reporting of Adverse Events (defined as any observation in an animal which was unfavorable and unintended and occurred after the use of either product) throughout the study period until D56. Blood samples for hematology and clinical chemistry were collected from all dogs at D0 prior to enrolment and at D28. Samples could be collected at other timepoints at the Investigator's discretion.

\section{Results}

\section{Study objects}

Thirty first opinion veterinary practices in France, Germany and the United Kingdom enrolled a total of 286 dogs in the study between April and September 2012. All dogs were considered for clinical safety, but one dog was excluded from all demographic and efficacy analyses as the Owner inadvertently retained both copies of the signed consent form. Demographics of the remaining 285 dogs (ITT population) are described herein. One hundred fifty-five males (51 neutered) and 130 female (68 neutered) were included for demographics and efficacy analyses. At the time of enrolment, dogs ranged in age from 10 weeks to 16.5 years with a mean $( \pm \mathrm{SD})$ of $6.0( \pm 3.8)$ years. The most common breeds represented were Labrador Retrievers (9\%), Cocker Spaniels (9\%), Golden Retrievers (6\%) and Cavalier King Charles Spaniels (6\%). Baseline demographics and disease characteristics are summarized by group in Table 2 .

There was no significant difference in any demographic or disease characteristic between groups.

As the results of the ITT and PP analyses were similar, only the results of the ITT analysis are listed and discussed below for efficacy related outcomes.

\section{Clinical evaluation}

The mean OTIS-3 and their standard deviations are listed in Table 3.

Table 2 Baseline Demographics

\begin{tabular}{llll}
\hline Demographic & Otic Gel & Otic Suspension & $P$-value \\
\hline Age in years (mean \pm SD) & $6.2 \pm 3.8$ & $5.8 \pm 3.7$ & 0.46 \\
Number of Male/Female (\%) & $55 / 45$ & $53 / 47$ & 0.72 \\
Weight in kg (mean \pm SD) & $21.9 \pm 14.4$ & $22.8 \pm 14.8$ & 0.62 \\
Bilateral otitis & $103(70 \%)$ & $101(74 \%)$ & 0.51 \\
Ceruminous exudate & $108(73 \%)$ & $96(70 \%)$ & 0.60 \\
Purulent exudate & $40(27 \%)$ & $41(30 \%)$ & \\
Acute/subchronic/chronic & $42(28 \%), 87$ & $30(22 \%), 90$ & 0.42 \\
& $(59 \%), 19(13 \%)$ & $(66 \%), 17(12 \%)$ & \\
Number of days since & $18.8 \pm 22.8$ & $18.6 \pm 22.5$ & 0.73 \\
onset (mean \pm SD) & & & \\
Previously treated & $13(9 \%)$ & $17(12 \%)$ & 0.34 \\
Recurrent & $46(31 \%)$ & $46(34 \%)$ & 0.90 \\
Culture yeast only (\%) & 25 & 27 & 0.89 \\
Culture bacteria only (\%) & 22 & 20 & \\
Culture yeasts and & 39 & 42 & \\
bacteria (\%) & & & \\
\hline
\end{tabular}


Table 3 Mean OTIS-3 \pm standard deviation of dogs treated with otic gel or otic suspension

\begin{tabular}{lllll}
\hline Criterion & Visit & $\begin{array}{l}\text { Otic gel } \\
(N=148)\end{array}$ & $\begin{array}{l}\text { Otic suspension } \\
(N=137)\end{array}$ & $P$-value \\
\hline OTIS-3 & D 0 & $6.8 \pm 1.6$ & $6.8 \pm 1.6$ & 0.7490 \\
& D 7 & $3.6 \pm 1.7$ & $3.0 \pm 1.9$ & 0.0027 \\
& D 28 & $2.6 \pm 2.2$ & $2.6 \pm 2.2$ & 0.7992 \\
& D 56 & $2.5 \pm 2.4$ & $2.7 \pm 2.4$ & 0.3532 \\
\hline
\end{tabular}

There was a significant improvement in OTIS-3 in both groups after 28 days $(p<0.0001)$. The mean OTIS-3 stayed low until D56 in both groups. The OTIS-3 decreased on average by 62.5 and $63.6 \%$ for the otic gel and by 63.4 and $60.5 \%$ for the otic suspension on D28 and D56 respectively. Using the non-inferiority margin of $15 \%$, the $95 \%$ confidence interval for the difference in percent reduction at D28 (otic suspension minus otic gel) had to be completely below $0.15 \times 63.4 \%$ i.e. $9.5 \%$. The Confidence Interval was calculated to be -6.1 to $7.9 \%$, so the primary endpoint of non-inferiority at D28 was concluded.

At D7, there was a significant difference between the two groups for total OTIS-3 $(p=0.0027)$ and the percentage reduction in OTIS-3 $(p=0.0003)$ in favour of the otic suspension. However, no significant difference was seen in the proportion of cases considered a clinical success by the Investigator at D7 (Table 4).

Approximately three quarters of clinicians and Owners reported a good to excellent response of otitis externa to the otic gel administration after D28 (Table 5), which was not significantly different to the suspension.

At D56, there were no differences between the group treated with the gel and that with the suspension in owners and investigators overall assessment scores. No significant differences in pain or pruritus scores were observed between the two groups at D28 or D56 ( $p>0.1860$; Table 6).

However, the owners reported a significant difference in pruritus at D7, in favor of the suspension $(p=0.0232)$. Relapses were recorded at D56 in 11 dogs (11\%) treated with the otic gel, and in 10 dogs (11\%) treated with otic suspension; there was no significant difference between the groups. Subsequent exploratory analyses did not identify any difference in clinical success at any timepoint between groups dependent on the weight of the $\operatorname{dog}(p>0.18$

Table 4 Number (Percentage) of dogs reported as clinical treatment success ${ }^{a}$ when treated with an otic gel or an otic suspension

\begin{tabular}{llll}
\hline Visit & Otic gel & Otic suspension & $P$-value \\
\hline D7 & $80(54 \%)$ & $84(61 \%)$ & 0.2319 \\
D 28 & $109(74 \%)$ & $98(72 \%)$ & 0.6926 \\
D 56 & $106(72 \%)$ & $91(66 \%)$ & 0.3705 \\
\hline
\end{tabular}

${ }^{a}$ Clinical success was defined as an OTIS- $3 \leq 3$
Table 5 Clinician (owner in parentheses) overall assessment of treatment response (in \%)

\begin{tabular}{lllll}
\hline & D 28 & \multicolumn{3}{c}{ D 56 } \\
\hline Response assessment & Gel & Suspension & Gel & Suspension \\
Excellent & $29(34)$ & $31(42)$ & $39(44)$ & $38(49)$ \\
Good & $45(49)$ & $40(42)$ & $32(36)$ & $28(24)$ \\
Moderate & $19(10)$ & $20(11)$ & $18(7)$ & $22(17)$ \\
Poor & $7(5)$ & $8(4)$ & $11(12)$ & $12(9)$ \\
Missing & $1(1)$ & $1(1)$ & $1(1)$ & $1(1)$ \\
p-value & \multicolumn{2}{c}{$0.9901(0.2692)$} & $0.6169(0.7980)$ \\
\hline
\end{tabular}

across all weight bands and time points), chronicity of disease $(p>0.1727)$, recurrence $(p>0.0947)$, previous treatment $(p>0.2025)$ or type of exudate $(p>0.0638)$.

\section{Microbiological evaluation}

On D0, 40\% of the overall study population had positive otic cultures for both bacteria and yeast, $26 \%$ had only yeast, $21 \%$ only bacteria and the remainder were culture negative; staphylococci of the Staphylococcus pseudintermedius group were most frequently cultured (47\%), followed by Pseudomonas aeruginosa (11\%), streptococci (11\%) and enterococci (5\%); in $18 \%$ of the dogs more than one bacterial species was identified. Of the proportion of the study population in which yeast was cultured, the most frequently identified organism was Malassezia pachydermatis (96\%). There was no difference in distribution between groups for either bacteria or yeast species at D0.

The cytology counts for bacteria, fungi and neutrophils decreased in both groups, with no significant difference seen between groups (Table 6).

The overall bacteriological response (i.e. elimination of pathogens identified at baseline culture) as assessed by repeat of bacterial culture, was $60 \%$ and $49 \%$ for the gel at D28 and D56 respectively, compared to 60\% and 55\% for the otic suspension. For the fungal response, $76 \%$ and $65 \%$ of cases treated with the otic gel had responded at D28 and D56 respectively, in comparison to 69\% and 55\% for the otic suspension. None of these were significantly different (Table 6).

When clinical success (OTIS-3 $\leq 3$ at D28) was considered for cases with different bacterial species, no significant differences were found between the products for any species. For $S$. pseudintermedius, the clinical success rate was $75 \%$ and $72 \%$ at D28 for the otic gel and the suspension respectively. For $P$. aeruginosa, the clinical success rates were $47 \%$ and $50 \%$ respectively for the otic gel and the otic suspension at D28.

\section{Minimum inhibitory concentrations}

The MIC data demonstrated that florfenicol was active against all bacterial groups, with $\mathrm{MIC}_{50}$ in the range 2 to 
Table 6 Mean scores of all dogs for cytology scores, microbiological responses, pain and pruritus scores

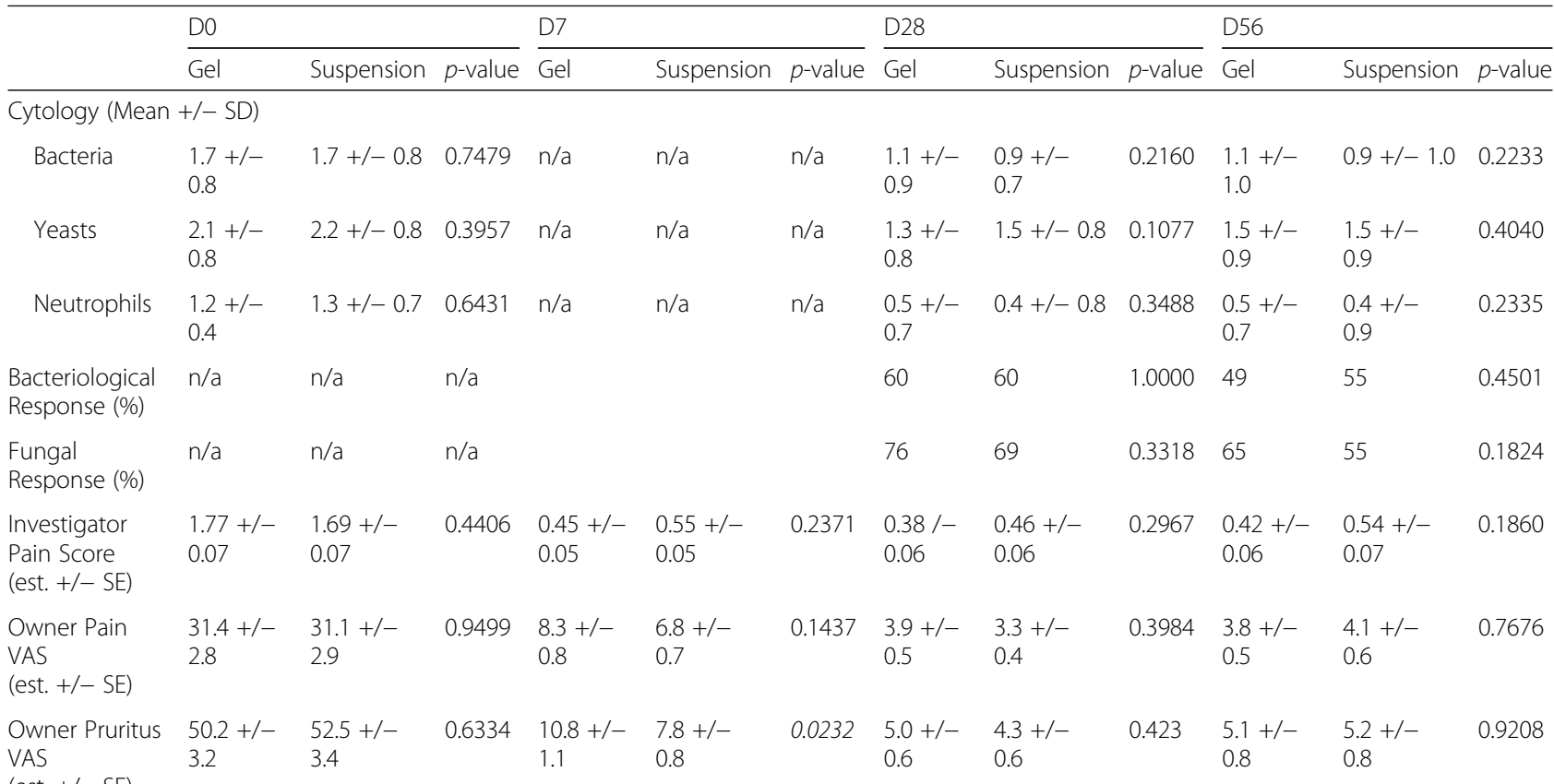

Est. Estimated, S.E. Standard error, VAS Visual analog scale, SD Standard deviation; Entries in italic font are statistically significant

$16 \mu \mathrm{g} / \mathrm{ml}$ except for P. aeruginosa $(>128 \mu \mathrm{g} / \mathrm{ml})$. Due to low numbers of isolates for other pathogens, the $\mathrm{MIC}_{90}$ could only be calculated for S. pseudintermedius $(8 \mu \mathrm{g} / \mathrm{ml})$, Streptococcus canis $(2 \mu \mathrm{g} / \mathrm{ml})$ and $P$. aeruginosa $(>128 \mu \mathrm{g} / \mathrm{ml})$. One hundred and two (102) S. pseudintermedius isolates were identified, of which 2 were identified as methicillin resistant on the basis of oxacillin zone diameter. Of 4 Staphylococcus aureus isolates, 2 were identified as methicillin resistant on the basis of cefoxitin zone diameter. All isolates identified as methicillin resistant exhibited florfenicol MICs within the same range as methicillin susceptible isolates of the corresponding species.

The only fungal species with greater than 10 isolates was $M$. pachydermatis. The MIC for terbinafine was in the range 0.125 to $>64 \mu \mathrm{g} / \mathrm{ml}$, with an $\mathrm{MIC}_{90}$ of $2 \mu \mathrm{g} / \mathrm{ml}$.

In 59\% of cases, there was no change in the MIC for florfenicol between baseline and D28 of any organism identified in an individual ear. In $28 \%$ of cases, there was an increase of MIC by one dilution, with a decrease by one dilution in the remainder (13\%). For terbinafine, there was no change in MIC between baseline and D28 for $55.5 \%$ of Malassezia cases, with $28 \%$ having an increase by one dilution, and $16.5 \%$ having a decrease by one dilution.

\section{Safety evaluation}

A total of 100 clinical signs relating to 80 adverse events were recorded in 30 of the dogs treated with the otic gel and 32 of the dogs treated with the suspension. These are summarized by System Organ Class in Table 7.
Cutaneous signs, such as generalized pruritus (rather than local to the ear) were the most common and observed in approximately one third of dogs showing adverse events, followed by slightly less dogs showing gastrointestinal signs. The majority of these adverse events were likely related to an underlying disease process such as atopic dermatitis. There was no difference in occurrence for any adverse event

Table 7 Clinical signs seen with administration of otic gel or suspension in dogs with otitis externa

\begin{tabular}{|c|c|c|c|}
\hline System Organ Class & $\begin{array}{l}\text { Otic Gel } \\
(n=148)\end{array}$ & $\begin{array}{l}\text { Otic Suspension } \\
(n=138)\end{array}$ & $P$-value \\
\hline Behavioral disorders & 0 & 2 & 0.2328 \\
\hline $\begin{array}{l}\text { Blood and lymphatic system } \\
\text { disorders }\end{array}$ & 3 & 0 & 0.2509 \\
\hline Digestive tract disorders & 15 & 10 & 0.4315 \\
\hline Ear and labyrinth disorders & 7 & 4 & 0.5516 \\
\hline Eye disorders & 1 & 6 & 0.0617 \\
\hline Hepato-biliary disorders & 1 & 0 & 1.0000 \\
\hline Mammary gland disorders & 0 & 1 & 0.4825 \\
\hline Musculoskeletal disorders & 1 & 3 & 0.3585 \\
\hline Neurological disorders & 1 & 0 & 1.0000 \\
\hline Renal and Urinary disorders & 0 & 1 & 0.4825 \\
\hline Reproductive system disorders & 0 & 1 & 0.4825 \\
\hline Respiratory tract disorders & 0 & 1 & 1.0000 \\
\hline Skin and appendages disorders & 16 & 18 & 0.6105 \\
\hline Systemic disorders & 5 & 2 & 0.4551 \\
\hline Any clinical sign & 51 & 49 & 0.9204 \\
\hline
\end{tabular}


between groups $(p=0.9204)$. There was no recognizable association between treatment and adverse events.

Three dogs treated with the otic gel showed clinically severe adverse events, none of those was considered to be related to medication due to the lack of temporal association and clinical signs observed. One dog had intestinal obstruction by peach stones 7 weeks after treatment, one a septic abdomen 6 weeks after treatment and the last one underwent exploratory surgery 7 days after inclusion in the study and showed gastrointestinal neoplasia. The first dog recovered uneventfully after surgery, the other two dogs were euthanized.

No changes in body weight, or clinically significant changes from baseline were seen in hematology or serum chemistry variables for either the gel or the suspension. There were no clinical pathology changes which could be considered of either hyper- or hypoadrenocorticism.

\section{Discussion}

In this study, in a population of dogs displaying typical characteristics of otitis externa, administration of an otic gel twice 1 week apart demonstrated similar efficacy and clinical safety to treatment with a conventional daily suspension for most endpoints. Only for the secondary endpoint speed of response (in terms of absolute and percentage reduction in OTIS-3 and Owner pruritus VAS) was the daily treatment significantly better than the otic gel. However, in another recently published study [9] using the otic gel, the percentage improvement in pruritus was considered to be better with the gel in comparison to a different daily treatment to that used in the current study. In that study, the comparator product contained a more potent corticosteroid (mometasone furoate) but had a smaller daily dose volume than the comparator in the current study. The smaller dose volume may not allow for sufficient contact between the product and the entire ear canal to rapidly reduce pruritus. Unlike the current study, Noli et al. [9] found that no difference in percentage improvement of OTIS-3 at D7 was seen between the gel and the comparator daily treatment. However, in that study, the gel provided significantly greater percentage improvements in cytology scores at Day 7 and Day 28. This suggests that the gel is capable of producing significantly faster responses to treatment than some daily treatments.

Approximately one third of dogs had recurrent otitis externa and in a little more than $10 \%$ the otitis was defined as chronic with those numbers comparatively lower than reported in one large epidemiological study [2]. This discrepancy may be explained by the fact that studies are frequently published by referral institutions and in contrast the practices involved in this study were first opinion. As in other studies, most dogs were presented with bilateral otitis, although the number of dogs with unilateral otitis externa was higher in this study than in reported studies (30\% versus $6-7 \%)[2,17]$, again probably reflecting the early presentation more typical for first opinion practice.

Staphylococci were the most common bacteria cultured from the dogs in this study, which is in accordance with other publications [17-20]. P. aeruginosa was cultured in a smaller number of dogs, also similar to other publications $[17,20]$. In contrast with other reports [21, 22], the smaller number of dogs infected with Pseudomonas in this study probably reflects early presentation and first opinion practice. A large number of dogs showed Malassezia organisms, either with or without concurrent bacteria. These findings are in concordance with other studies [2, 3, 17], although in most publications, the exact number of dogs showing Malassezia organisms only versus concurrent bacterial and yeast infection was not stated.

The OTIS-3 scale used in this study was validated in a recent study [16]. A total score of $\geq 4$ differentiated healthy from diseased ears with a specificity of $100 \%$ and a sensitivity of over $90 \%$. Based on that publication, an OTIS-3 $\geq$ 5 was a criterion of inclusion in this study and an OTIS-3 of $\leq 3$ was considered an ear in clinical remission. Utilizing this score, slightly less than three quarters of the dogs included in this study were in clinical remission after two treatments with the gel and approximately two thirds after five treatments with the suspension. This compares favorably to a number of other studies. In a study using a zinc-acetic acid ear cleaner for 14 days, $25 \%$ of the dogs with otitis externa due to $M$. pachydermatis had a clinical score indicating clinical remission [23]. In another study, 140 dogs were treated with either a marbofloxacin/dexamethasone/clotrimazole suspension daily or with miconazole/polymixin $\mathrm{B} /$ prednisolone daily for 7 to 14 days. The cure rate by day 14 was 58 and $41 \%$ respectively, defined by absence of erythema or ulceration, pruritus, pain and smell [24]. The latter study was also conducted in first opinion practices. A different method of scoring was used in each of these 3 studies making direct comparison difficult. One small study treating 12 dogs with twice daily ear cleaner and ticarcillin for up to 1 month had a better success rate, all but one dog responded well [25]. However, this study was not controlled, the dogs were referred for chronic otitis and treated much more aggressively including repeated procedures of ear flushing under anaesthesia. Owners used an ear cleaner twice daily and the ear medications were used for more than 2 weeks.

Cytology of the ear swabs showed a significant reduction of yeast and bacteria during treatment in both groups. On D28, approximately two thirds of the population and on D56, approximately half of the population showed no or few bacteria cytologically. Only a few studies have evaluated cytology subsequent to the treatment of otitis externa, and the methods differed in each study, thus comparisons are difficult. In a study using a 
zinc-acetic acid ear cleaner for 14 days, $58 \%$ of the dogs with otitis externa due to $M$. pachydermatis had few or no yeast after 14 days [23]. In a study of dogs with Malassezia otitis treated with either miconazole/ dexamethasone/saline or the same solution with an added chelating agent the clinical scores and cytological scores for yeast organisms decreased significantly, but it was not stated how many dogs were negative on cytology and in remission clinically [26]. The apparent increase in bacterial cytological counts between D28 and D56 is likely to be as a result of recolonization of the ear canal by the normal flora.

Determination of MICs in this study demonstrated that most routinely identified pathogens associated with otitis externa are highly susceptible to both florfenicol and terbinafine. It was noted that despite the higher MIC, the clinical success rate in cases with $P$. aeruginosa, whilst lower than clinical success rates for S. pseudintermedius, was similar in both groups at D28. This is likely because topically applied antibiotics will more often than not exceed MICs established for systemic administration, even for apparently resistant organisms [27]. Although changes in MIC were observed in approximately $40 \%$ of cases at D28 compared to baseline, these were never by more than one dilution. It is considered that this is due to normal variation in the reproducibility of MIC data, as the changes were in both directions from baseline. Thus, despite the persistence of the antimicrobials within the ear canal [28], no evidence of a change in susceptibility of the target organisms was observed in this study.

Adverse events were seen in approximately $15 \%$ of the dogs in this study. However, it is important to note that adverse events were classified as any unfavorable and unintended event during or after administration of the medications, whether or not considered to be product related [29]. Adverse events by and large could be divided into two major groups. Firstly, clinical signs such as pruritus, dermatitis, conjunctivitis, otitis, or pyoderma which were most likely related to the primary cause of the otitis externa; and secondly clinical signs such as vomiting or diarrhoea, which were unlikely to be related to the underlying cause of otitis. Half of all dogs with allergic skin disease are presented with otitis externa $[4,30]$. Allergic skin disease frequently waxes and wanes depending on the offending allergens and is associated with pruritus and secondary infections $[4,30]$, thus it is not unreasonable to assume that increased generalized pruritus in a patient is more likely due to its underlying allergy than due to the application of an otic topical. It was not the goal of the study to determine the underlying predisposing causes and as such no detailed history of other clinical signs was recorded, which would have been useful to determine whether indeed these adverse events were previously present. The second large group of adverse events included signs such as abdominal pain, pseudopregnancies, arthritis and vomiting. These clinical signs were most likely not related to the otitis externa, its primary cause or its treatment. Arthritis is a common complaint of older dogs [31] and the age of dogs included in this study was up to 16 years with a mean of 6.0 years. Gastrointestinal signs such as vomiting and diarrhea are frequently seen in dogs [32,33], their cause is often unknown and they often resolve without specific therapy. Only three dogs (all treated with the otic gel) were withdrawn from the study due to serious adverse events, which were gastrointestinal neoplasia, obstruction due to intestinal foreign bodies and a septic abdomen 6 weeks after enrolment. These are highly unlikely to be related to the treatment of the otitis externa. Overall, adverse effects were considered not to be related to treatment, but rather to underlying or concurrent diseases and no clinically relevant changes were seen in any serum biochemistry or hematology parameter.

\section{Conclusion}

The non-inferiority of the otic gel to the otic suspension as a reference product was demonstrated following two administrations of the gel at a one-week interval. Clinical signs of the dogs with otitis externa improved similarly in the two groups, independent of the nature and chronicity of the infection. In both groups there was a significant decrease in otic scores and microorganisms as well as a rapid improvement in pain and pruritus scores. The otic gel was well tolerated in a wide range of ages and breeds of dog and thus offers a safe and effective treatment alternative for dogs with otitis externa with the added benefit of requiring only two administrations 1 week apart, providing the potential for improved compliance with the treatment regimen.

\section{Abbreviations}

ANCOVA: Analysis of covariance; CLSI: Clinical and Laboratory Standards Institute; D: Day; ITT: Intent to treat; MIC: Minimum inhibitory concentration; OTIS-3: Otitis index score; PP: Per protocol; SD: Standard deviation

\section{Acknowledgements \\ The authors would like to thank the participating veterinarians and pet owners. \\ Funding \\ The study was funded by Novartis Animal Health which is now owned by Elanco Animal Health; this includes design of study, and collection, analysis, and interpretation of data, and writing of manuscript.}

\section{Availability of data and materials}

All data cannot be freely shared without control due to confidentiality. Please contact the corresponding author to request access to nonconfidential data.

\section{Authors' contributions}

SBK and SLF were involved in the study design, in-life phase of the study, and interpretation of results and writing of the manuscript. KPD was involved in the interpretation of results and writing of the manuscript. WS was responsible for data analysis, and review of the manuscript. All authors contributed to later versions and agreed with the manuscript. 


\section{Ethics approval}

The study was conducted in accordance with guidelines for Good Clinical Practice (VICH GL9). The protocol was reviewed and approved by Novartis Animal Health Inc.'s (acquired by Elanco Animal Health,) Institutional Anima Care and Use Committee. All owners provided written consent upon their dog entering the study (Day 0).

\section{Consent for publication}

Not applicable.

\section{Competing interests}

All authors are employees of Elanco Animal Health.

\section{Publisher's Note}

Springer Nature remains neutral with regard to jurisdictional claims in published maps and institutional affiliations.

\section{Author details}

'Elanco Animal Health, 2500 Innovation Way, Greenfield, IN 46140, USA. ${ }^{2}$ Elanco Animal Health Inc., Mattenstrasse 24a, CH-4058 Basel, Switzerland. ${ }^{3}$ Elanco Animal Health Ltd., Lilly House, Priestley Road, Basingstoke, Hampshire RG24 9NL, UK.

Received: 11 December 2017 Accepted: 25 September 2018 Published online: 11 October 2018

\section{References}

1. August JR. Otitis externa: a disease of multifactorial etiology. Vet Clin North Am Small Anim Pract. 1988;18:731-42.

2. Saridomichelakis MN, Farmaki R, Leontides LS, et al. Aetiology of canine otitis externa: a retrospective study of 100 cases. Vet Dermatol. 2007;18:341-7.

3. Zur G, Lifshitz B, Bdolah-Abram T. The association between the signalment, common causes of canine otitis externa and pathogens. J Small Anim Pract. 2011;52:254-8

4. Griffin CE, DeBoer DJ. The ACVD task force on canine atopic dermatitis (XIV): clinical manifestations of canine atopic dermatitis. Vet Immunol Immunopathol. 2001;81:255-69.

5. Angus JC. Otic cytology in health and disease. Vet Clin North Am Small Anim Pract. 2004;34:411-24.

6. Nuttall T, Cole LK. Evidence-based veterinary dermatology: a systematic review of interventions for treatment of Pseudomonas otitis in dogs. Vet Dermatol. 2007:18:69-77.

7. Boda C, Liege P, Reme CA. Evaluation of owner compliance with topical treatment of acute otitis externa in dogs: a comparative study of two auricular formulations. Int J Appl Res Vet Med. 2011;9:157-65.

8. Miller WH, Griffin CE, Campbell KL. Diseases of the eyelids, claws, anal sacs and ears. In: Miller WH, Griffin CE, Campbell KL, editors. Muller \& Kirk's small animal dermatology. 7th ed. St. Louis: Elsevier; 2013. p. 724-73.

9. Noli C, Sartori R, Cena T. Impact of a terbinafine-florfenicol-betamethasone acetate otic gel on the quality of life of dogs with acute otitis externa and their owners. Vet Dermatol. 2017. https://doi.org/10.1111/vde.12433.

10. Beck KM, Waisglass SE, Dick HL, et al. Prevalence of meticillin-resistant Staphylococcus pseudintermedius (MRSP) from skin and carriage sites of dogs after treatment of their meticillin-resistant or meticillin-sensitive staphylococcal pyoderma. Vet Dermatol. 2012;23:369-75.

11. Loeffler A, Boag AK, Sung J, et al. Prevalence of methicillin-resistant Staphylococcus aureus among staff and pets in a small animal referral hospital in the UK. J Antimicrob Chemother. 2005;56:692-7.

12. Sasaki T, Kikuchi K, Tanaka Y, et al. Methicillin-resistant Staphylococcus pseudintermedius in a veterinary teaching hospital. J Clin Microbiol. 2007; 45:1118-25.

13. van Duijkeren E, Kamphuis M, van der Mije IC, et al. Transmission of methicillin-resistant Staphylococcus pseudintermedius between infected dogs and cats and contact pets, humans and the environment in households and veterinary clinics. Vet Microbiol. 2011;150:338-43.

14. Andersson DI, Hughes D. Microbiological effects of sublethal levels of antibiotics. Nat Rev Microbiol. 2014;12:465-78.

15. Sandegren L. Selection of antibiotic resistance at very low antibiotic concentrations. Ups J Med Sci. 2014;119:103-7.
16. Nuttall T, Bensignor E. A pilot study to develop an objective clinical score for canine otitis externa. Vet Dermatol. 2014;25:530-7 e591-532.

17. Yoshida N, Naito F, Fukata T. Studies of certain factors affecting the microenvironment and microflora of the external ear of the dog in health and disease. J Vet Med Sci. 2002;64:1145-7.

18. Bouassiba C, Osthold W, Mueller RS. Comparison of four different staining methods for ear cytology of dogs with otitis externa. Tierarztl Prax Ausg K Kleintiere Heimtiere. 2013;41:7-15.

19. Degi J, Imre K, Catana N, et al. Frequency of isolation and antibiotic resistance of staphylococcal flora from external otitis of dogs. Vet Rec. 2013;173:42.

20. Oliveira LC, Leite CA, Brilhante RS, et al. Comparative study of the microbial profile from bilateral canine otitis externa. Can Vet J. 2008;49:785-8.

21. Hariharan $\mathrm{H}$, Coles M, Poole D, et al. Update on antimicrobial susceptibilities of bacterial isolates from canine and feline otitis externa. Can Vet J. 2006;47:253-5.

22. Bugden DL. Identification and antibiotic susceptibility of bacterial isolates from dogs with otitis externa in Australia. Aust Vet J. 2013;91:43-6.

23. Mendelsohn CL, Griffin CE, Rosenkrantz WS, et al. Efficacy of boriccomplexed zinc and acetic-complexed zinc otic preparations for canine yeast otitis externa. J Am Anim Hosp Assoc. 2005;41:12-21.

24. Rougier S, Borell D, Pheulpin S, et al. A comparative study of two antimicrobial/anti-inflammatory formulations in the treatment of canine otitis externa. Vet Dermatol. 2005;16:299-307.

25. Nuttall TJ. Use of ticarcillin in the management of canine otitis externa complicated by Pseudomonas aeruginosa. J Small Anim Pract. 1998;39:165-8.

26. Hensel $\mathrm{P}$, Austel $\mathrm{M}$, Wooley RE, et al. In vitro and in vivo evaluation of a potentiated miconazole aural solution in chronic Malassezia otitis externa in dogs. Vet Dermatol. 2009;20:429-34.

27. Ohyama M, Furuta S, Katsuda K, Nobori T, Kiyota R, Miyazaki Y. Ofloxacin otic solution in patients with otitis media: an analysis of drug concentrations. Arch Otolaryngol Head Neck Surg 1999;125(3):337-340.

28. Nuttall T, Forster S. Terbinafine and florfenicol concentrations in the canine ear canal exceed minimum inhibitory concentrations for common otic pathogens after treatment with Osurnia ${ }^{\circledast}$ (Elanco Animal Health). In: Proceedings of the British Veterinary Dermatology Study Group spring meeting, Birmingham, UK; 2015. p. 91-4.

29. VICH. Pharmacovigilance of veterinary medicinal products: management of adverse event reports. In: (VICH) International Cooperation on Harmonisation of Technical Rrequirements for Registration of Veterinary Medicinal Products, ed. Bruxelles: VICH GL 24 (Pharmacovigilance: AERs); 2007.

30. Saridomichelakis MN, Koutinas AF, Gioulekas D, et al. Canine atopic dermatitis in Greece: clinical observations and the prevalence of positive intradermal test reactions in 91 spontaneous cases. Vet Immunol Immunopathol. 1999;69:61-73.

31. Caron-Lormier G, England GC, Green MJ, et al. Using the incidence and impact of health conditions in guide dogs to investigate healthy ageing in working dogs. Vet J. 2016;207:124-30.

32. Lund EM, Armstrong PJ, Kirk CA, et al. Health status and population characteristics of dogs and cats examined at private veterinary practices in the United States. J Am Vet Med Assoc. 1999:214:1336-41.

33. Summers JF, O'Neill DG, Church DB, et al. Prevalence of disorders recorded in Cavalier King Charles Spaniels attending primary-care veterinary practices in England. Canine Genet Epidemiol. 2015;2:4.

Ready to submit your research? Choose BMC and benefit from:

- fast, convenient online submission

- thorough peer review by experienced researchers in your field

- rapid publication on acceptance

- support for research data, including large and complex data types

- gold Open Access which fosters wider collaboration and increased citations

- maximum visibility for your research: over $100 \mathrm{M}$ website views per year

At BMC, research is always in progress.

Learn more biomedcentral.com/submissions 